International Journal of Engineering \& Technology, 7 (4.20)(2018) 157-161
International Journal of Engineering \& Technology
SPC
Website: www.sciencepubco.com/index.php/IJET
Research paper

\title{
Prediction the Behavior of Piles in Clayey Soils
}

\author{
Hanan Adnan Hasan* \\ Water Resource department/Faculty of Engineering/Al-Mustansiria University/Iraq-Baghdad \\ *Corresponding author E-mail: ghassan_hanan@yahoo.com.
}

\begin{abstract}
This research includes a non- linear finite element analysis of axisymmetric soil-pile system. The analysis included piles of different length and diameter. The interface element was used as a thin layer separating the pile from surrounding soil. The load settlement curves for piles installed in soft to medium and stiff clays were obtained. Two consideration were studied; the ultimate capacity of pile and the settlement reduction factor. The results show that increase in the length and diameter of pile leads to increase in the ultimate load in range (35-60\%) and in the range (33-35) \% respectively. The distribution of lateral displacement along the depth of the pile gave the same trend in both soft to medium and stiff clay while the settlement reduction factor in soft soil is greater than for the stiff clay.
\end{abstract}

Keywords: Clayey Soil; Finite Element; Interaction; Piles; Settlement Curve; Ultimate Bearing Capacity.

\section{Introduction}

It is usually have a relatively small cross- sectional dimension with respect to their length. Piles derive their carrying capacity from end bearing of their bases and/ or the friction forces acting on their surface area.

Soft soil deposits of low shear strength and high compressibility are found worldwide. In the past, construction on these deposits was avoided because of their adequacy in as a foundation ground due to their low bearing capacity to support buildings, road, etc.

The pile foundation system functions properly only when both components (the soil and the pile) support loads without failure or excessive settlement depends on number of factors such as: pile characteristics, soil profile, soil properties, loading on the pile, type of loading and direction of loading. Most of the existing methods of pile design start with estimating the axial capacity or the load- settlement behavior of a single pile (Poulos, 1987).

In this study, an attempt has been made to investigate the influence of length, diameter of pile and change in soil properties on the ultimate capacity of pile and the settlement reduction factor through the application of finite element (CRISP) software program.

\section{Soil-pile interaction}

Load on rigid piles is primarily resisted by shear stresses developed on the interface and a small amount by bearing at the pile base. The system behavious linearly up to a certain stage of loading, but with further increase in load, the interface develops a zone of local shear failure and the system begins to deviate from linearity. The load transferred to base increases simultaneously until full mobilization of shaft occurs. In the case of deformable piles when the displacement at the pile head is much large than that of pile base forming slip (or yield) in the upper portion of the pile while the lower portion is still in the elastic range. This requires model between the pile and the soil that relates the load and displacement instead of the tradional soil model relating stress and strain through the spring model (Poulos and Davis, 1980).

Settlement Analysis of Piles

The techniques for settlement investigation might be grouped into three general classes:

1. Load-exchange techniques, in which estimated connections between heap opposition and heap development at different focuses along the heap are utilized.

2. Methods dependent on the hypothesis of flexibility that utilize the conditions of Mindlin (1936) for subsurface stacking inside a semi-interminable mass.

3. Numerical techniques, and specifically, the limited component strategy.

Meyerhof suggested that at loads less than about one third of the ultimate, the settlement $(\mathrm{P})$ of pile could be estimated as follows (provided that no softer layers exist beneath the pile):

$P=\frac{D_{b}}{30 F}$

Where:

$\mathrm{D}_{\mathrm{b}}=$ diameter of pile base

$\mathrm{F}=$ factor of safety $(>3)$ on ultimate load.

Focht (1967) examined data from a number of load tests and related the observed settlement, $\mathrm{P}$ at the working load to the computed column deformation, $\mathrm{P}_{\mathrm{col}}$, at the working load.

The advantage of the finite element method over other numerical methods is that it can be systematically programmed to accommodate such complex and difficult material properties which usually occur in soils such as, non homogeneous, anisotropic, non-linear, hysteretic time- dependent, as well as difficult boundary conditions. Soil stratification easily be incorporated in this method. 
Many investigations have employed the finite element method to analyze the behavior of this method, and its versatility and efficiency in solving pile-soil interaction problems; it is used in this research (Al-Ani, 2001, Yang and Jeremic,2003, Ashour, 2004).

\section{Modeling the Problem}

For linear analysis, the finite element technique is widely employed as a design tool. Similar acceptance of non-linear situations depends on two major factors: first, considerable computing power is required in view of the increased numerical operations associated with non-linear problems. Secondly, before the finite element method can be used in design, the accuracy of any proposed solution technique must be proved. Loading condition for a threedimensional solid which is symmetrical about its centerline axis and subjected to loads and boundary condition that are also symmetrical about this axis, shows behavior independent of the circumferential rotation (Toma, 2010).

In the analysis, the pile is treated as an axisymmetric rotation case. The problem can be discretized into 4-node isoparametric quadrilateral elements. Each node in the element has two degrees of freedom to describe the vertical and horizontal displacements, the nodal displacements were defined by (Smith and Griffiths, 1988, Abu-Farsakh, 2011)

$$
\left\{\delta_{i}\right\}=\left(\begin{array}{c}
u_{i} \\
v_{i}
\end{array}\right)
$$

Where:

$u i=$ displacement in $\mathrm{X}$ direction

$v i=$ displacement in $\mathrm{Z}$ direction

The element displacement by the vector:

$\{\delta\}^{e}=\left(\begin{array}{c}\delta_{1} \\ \delta_{2} \\ \delta_{3} \\ \delta_{4}\end{array}\right)$

Thus, the displacement at any point within the element will be:

$$
\{u\}=\left(\begin{array}{c}
u \\
v
\end{array}\right)=\{N\}\{\delta\}^{e}
$$

where:

$\{u\}=$ the displacement vector.

$\{N\}=$ the shape function vector of the element.

The strain vector defines in terms of the displacement of that point.

$\{\varepsilon\}=\left(\begin{array}{c}\varepsilon_{r} \\ \varepsilon_{z} \\ \boldsymbol{\varepsilon}_{\boldsymbol{\theta}} \\ \gamma_{r z}\end{array}\right)$

Where:

$\{\varepsilon\}=$ the strain vector.

$\varepsilon r, \varepsilon_{z}, \varepsilon \theta=$ the strain in $\mathrm{r}, \mathrm{z}, \theta$-directions respectively.

$\gamma_{r z}=$ the shear strain in the rz-plane.

Using the displacement function, defined by Eq.(2) the strain displacement relationship can be written as follows

$$
\{\varepsilon\}=[B]\{\delta\}^{e}
$$

$[B]=$ the strain-displacement matrix.

$$
[B]=[A][N]
$$

The stress-strain relationship is given as:

$$
\{\sigma\}=[D]\{\varepsilon\}
$$

Where:

$\{\sigma\}=$ the stress tensor.

$[D]=$ the stress-strain matrix.

The element stiffness equation is:

$$
K M=\iiint B^{t} D B r d r d z d \theta
$$

When integrated over one radian becomes:

$$
K M=\iint B^{t} D B r d r d z d \theta
$$

Where the superscript (t) refers to transpose of a matrix. The element stiffness matrix of all element is assembled to obtain the global stiffness matrix $[\mathrm{K}]$ for the whole structure (Smith and Griffiths,1988).

During the past years special interface elements have been developed to simulate the relative movement or slip at the soilstructure interface. The importance of interaction phenomenon in static and dynamic soil-structure interaction was recognized and studied by many investigators (Al-Ani, 2001).

Goodman et. al.(1968) were the first to propose utilizing a joint component to break down the conduct of shake joints. This component is a 4-hub component of length $\mathrm{L}$ and zero thickness (i.e., hubs 1, 2 and 3, 4 are incidental at first). The component dislodging was expected to differ directly between the hubs.

Desai et. al. (1984) pushed the utilization of the thin layer component in soil-structure communication and shake joints. Different twisting modes, for example, no slip, slip, debonding and rebonding as appeared in Figure 1, were utilized. The proposed component basically speaks to a strong component of little limited thickness or a thin layer of material between two bodies.
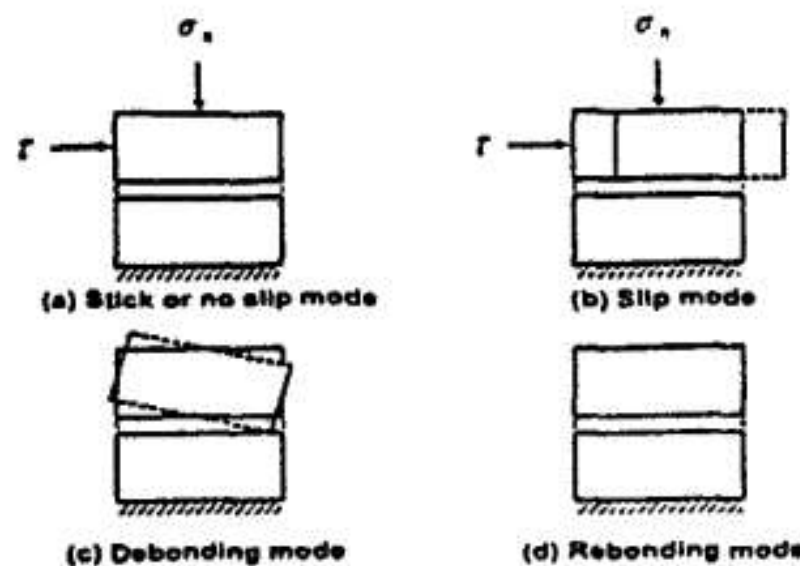

Fig.1: Schematic diagram of modes of deformation at interface

$$
\{d \sigma\}=] D]\{d \varepsilon\}
$$

Where:

$\left[\overline{D_{n}}\right]=$ normal behavior of the thin interface element.

Superscripts g and $\mathrm{st}=$ geological and structural materials respectively.

$\lambda_{1} . \lambda_{2}$ and $\lambda_{3}=$ the participation factors which mav vary from 0 to

$$
\left[D_{n}\right]=\lambda_{1}\left[D_{n}\right]_{i}+\lambda_{2}\left[D_{n}^{g_{n}}\right]+\lambda_{3}\left[D^{s t}{ }_{n}\right]
$$

The interface element in this research depends on the stiffness matrix of only normal and shear stress in the axisymmetric interface domain and zero thickness. For soil-structure interaction problems, it is appropriate to assume that the behavior near the interface involves a finite thin zone rather than zero thickness and 
this interface assumption can provide good or improved computations.

The choice of thickness is an important question and can be resolved by performing parametric studies in which the predictions from various thicknesses are compared with observation.

As per parametric examination including limited component counts and research center shear test on sand-solid interface, it was discovered that attractive recreation of interface conduct can be gotten for $\mathrm{t} / \mathrm{B}$ (where $\mathrm{t}$ is the thickness of interface and $\mathrm{B}$ is the length of the thin layer component) proportions in the range from 0.01 to 0.1 (Desai et. al. 1984). In the limited component strategy, the area of the issue including heap and diverse kinds of soils is descretized into number of soil and heap components. The solidness of every component characterized by utilizing the constitutive connection for the component material, the heap material whose quality is normally a lot higher than of the encompassing soil, is thought to be direct elastic(Desia,1977). The dirt is by and large non-straight material and ought to be dealt with along these lines, the modulus of versatility of strengthened cement is regularly taken as equivalent to $2000 \mathrm{Mpa}$, the toxins proportion is equivalent to 0.15 and the join weight is equivalent to $25 \mathrm{kN} / \mathrm{m} 3$.

The tangent modulus $\left(\mathrm{E}_{\mathrm{t}}\right)$ at any level of stress and strain was given by Duncan and Chang (1970) as:

$$
E_{t}=E_{i}\left(1-\frac{R_{f} \sigma}{S}\right)
$$

$E_{i}=$ the initial tangent modulus.

$f=$ a parameter obtained from fitting procedure using the hyperbolic model of Kondner (1963).

The initial modulus is found to vary with the confining pressure according to:

$$
E_{i}=K \cdot p a\left(\frac{\sigma_{3}}{p a}\right)^{\mathrm{n}}
$$

Where:

$\mathrm{Pa}=$ the atmospheric pressure.

$\mathrm{K}, \mathrm{n}=$ constant .

The complete relation then becomes:

$$
E_{i}=K \cdot p a\left(\frac{\sigma_{3}}{P a}\right)^{n} 1-\left(\frac{R_{f}\left(\sigma_{l}-\sigma_{3}\right)(1-\sin \phi)}{2 \sigma_{3} \sin \phi+2 C \cos \phi}\right)
$$

The parameters $R_{f}, \mathrm{~K}$ are determined experimentally.

Desai (1977) presented the hyperbolic model to represent the non-linear behavior of interface at prepeak. The tangent shear stiffness $\mathrm{K}_{\text {st }}$ at any stress level is computed as follows:

$$
K_{s t}=K_{s i} 1-\left(R_{f} \bar{S}\right)^{2}
$$

Where:

$$
\begin{aligned}
& \mathrm{K}_{\mathrm{si}}=\mathrm{K}_{\mathrm{j}} \gamma_{\mathrm{w}}\left(\sigma_{\mathrm{n}} / \mathrm{pa}\right)^{\mathrm{n}} \\
& \overline{\mathrm{S}}=\tau / \tau_{f}
\end{aligned}
$$

Where: $K_{\text {si }}$ is the initial tangent shear stiffness, $\gamma_{\mathrm{w}}$ is the unit weight of water in the same units as $\mathrm{K}_{\mathrm{si}}, \mathrm{S}$ is the shear stress level, $\tau$ and $\tau_{f}$ are the shear stress and shear stress at failure respectively, $\mathrm{K}_{\mathrm{j}}, \mathrm{n}, R_{f}$ are curve fitting parameter determined from a set of $\tau-u$ curves for different normal stresses.

\section{Description of the Problem}

The problem consists of a single pile embedded in clay layer. Two types of clays are studied, soft to medium clay and stiff clay. The finite element mesh used in the analysis is shown in figure (2) and material properties adopted in the analysis are given in table (1).

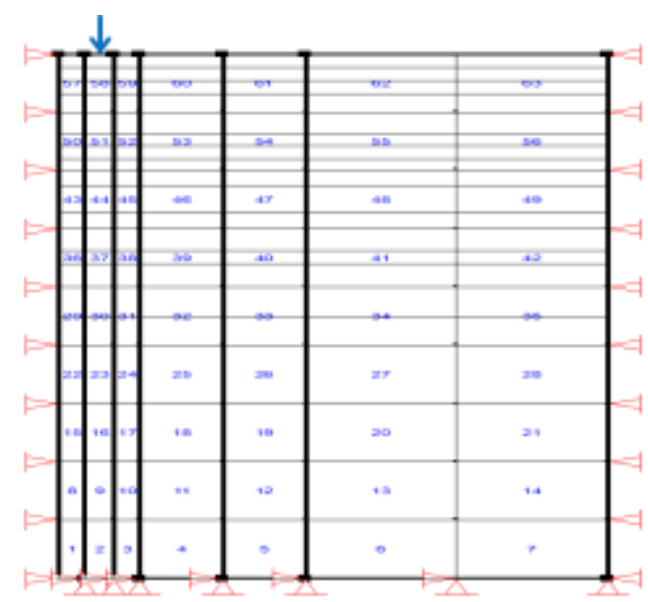

Fig. 2: Typical Finite Element Mesh

Table 1: Properties of Material

\begin{tabular}{cccc}
\hline Type of clay & Soft to medium & stiff \\
\hline$v$ & & 0.49 & 0.4 \\
$\phi^{\circ}$ & & 5 & 10 \\
$\mathrm{C}_{\mathrm{u}}\left(\mathrm{kN} / \mathrm{m}^{2}\right)$ & 25 & 75 \\
$\mathrm{E}(\mathrm{MPa})$ & & 3 & 15 \\
$\gamma\left(\mathrm{kN} / \mathrm{m}^{3}\right)$ & & 18 & 19 \\
$\mathrm{E}_{\mathrm{t}}$ & $\mathrm{N}$ & 0.65 & 0.5 \\
parameters & $\mathrm{K}$ & 150 & 300 \\
& $\mathrm{R}_{\mathrm{f}}$ & 0.93 & 0.9 \\
$\mathrm{~K}_{\mathrm{s}}$ & $\mathrm{n}$ & 0.315 & 0.315 \\
parameters & $\mathrm{K}_{\mathrm{j}}$ & 800 & 2000 \\
& $\mathrm{R}_{\mathrm{f}}$ & 0.83 & 0.83 \\
$\delta$ & & 7 & 35
\end{tabular}

The computer finite element program is a group of subroutine adopts several types of elements such as quadrilateral, triangular, bar and, interface elements. The program is capable of solving three types of problem, i.e., plane-strain, plane-stress and axisymmteric. The problem solved can model linear-elastic or nonlinear material behavior.

The problem domain will be solved by the finite element method and boundary condition; due to the symmetry of loading as well as the geometry the depth of this half is $20 \mathrm{~m}$ and the width is $10 \mathrm{~m}$. The nodal points along the centerline and those on the right vertical boundary are constrained to move only in the vertical direction, where as those on the bottom boundary are totally fixed. The rest of the nodal boundary points; those at the surface, are constrained as shown in figure (2).

The pile-soil interaction is molded through the interface element and the initial stresses are calculated.

The pile and the soil are represented by four nodal isoparametric elements, while the interface regions between the pile and the soil are represented by thin-layer interface elements.

The numbering of the nodal points must be made in such a way that the difference between any two nodal points belonging to any elements is minimum in order to minimize the band width of the total stiffness matrix $[\mathrm{K}]$ and hence the solution time. Accordingly, the numbering of the nodal points is made along the short dimension of the mesh.

The pile is loaded by equal increments of $300 \mathrm{kN}$ and the loading is continued until the pile failure occurs. The failure is defined according to the maximum settlement criterion, i.e. $10 \%$ of pile diameter. 


\section{Result and Analysis}

\subsection{Effect of pile length}

The figure (3) indicates that the load-settlement curves have the same shape for different lengths. An increase in the ultimate load range (35-60) \% can be obtained when the pile length is increased from $(6 \mathrm{~m})$ to $(14 \mathrm{~m})$

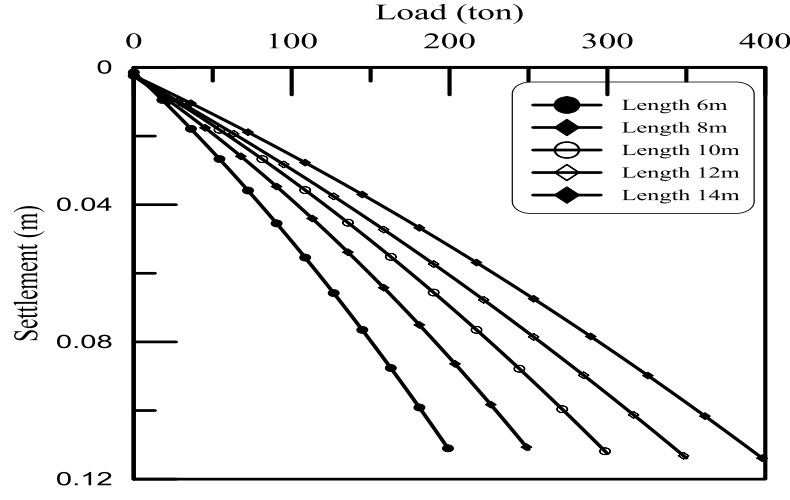

a. Soft to medium soil

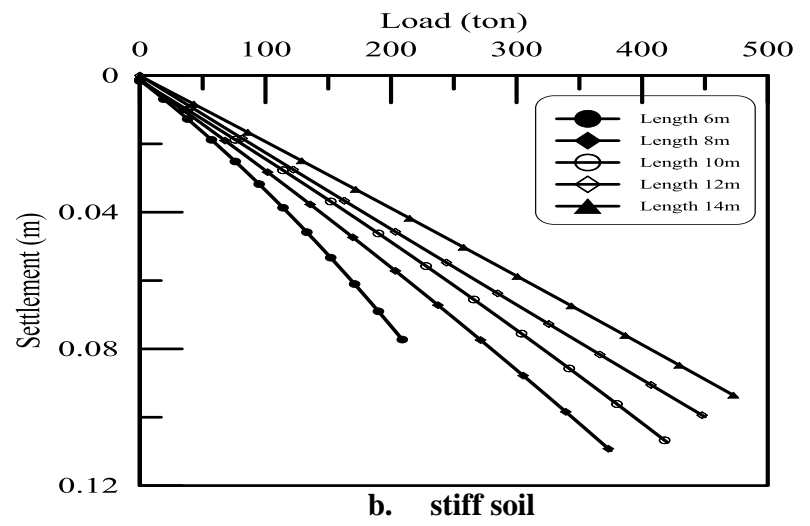

Fig. 3: Effect of pile length on load- settlement curves (pile diameter $=1.0 \mathrm{~m})$

\subsection{Effect of pile diameter}

The figure (4) observed that an increase in the ultimate load of the pile in the range (33-35) \% can be obtained when the pile diameter is increased from (1.2) $\mathrm{m}$ to $(2.0) \mathrm{m}$.

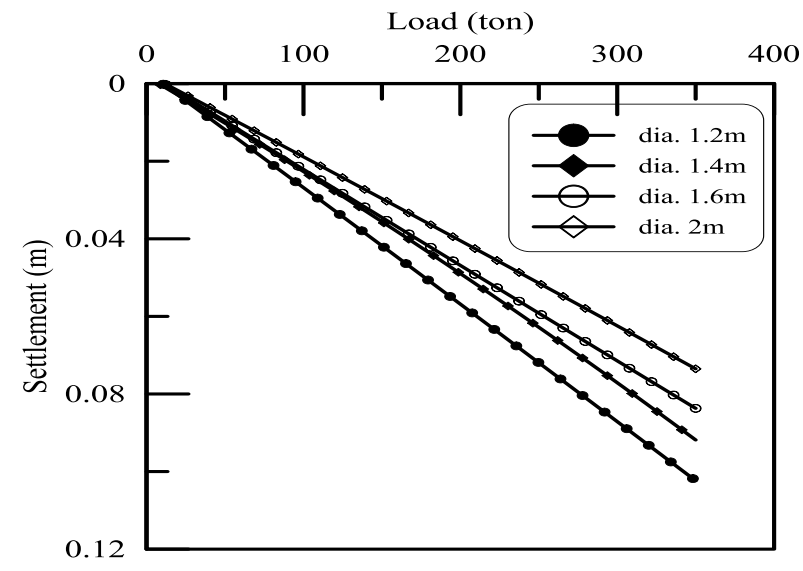

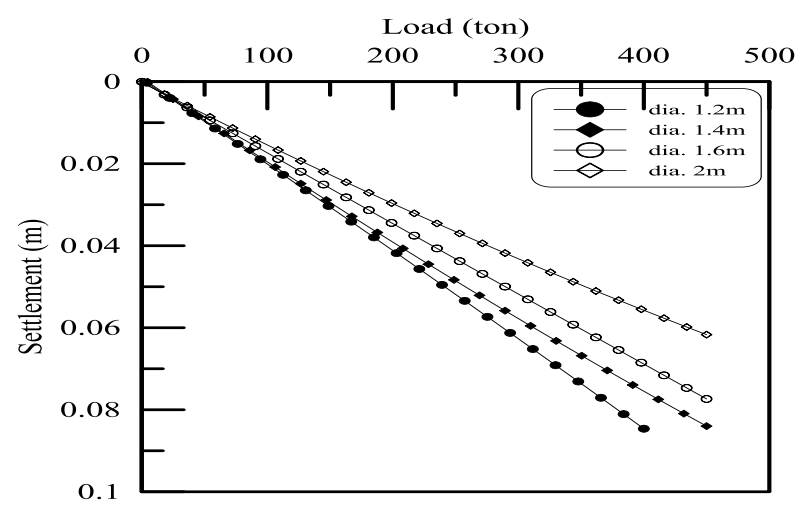

b. stiff soil

Fig. 4: Effect of pile diameter on load- settlement curves (pile length=10m)

\subsection{Settlement reduction factor}

In order to study the effect of pile in reducing the settlement of the clay layer under the

$$
\text { Settlement reduction factor }=\frac{\text { Settlement of (pile soil) system }}{\text { Settlement of untreated soil }}
$$

Figure (5) shows the distribution of the settlement reduction factor with depth in soft and stiff clays for different length of piles. It's noticed that there is nearly a constant load (70 ton) which separates the effect of soil strength. Below this load, the settlement reduction factor for the soft clay is greater than for the stiff clay. Figure (6) shows the distribution of lateral displacement with depth under a load of 150 ton. It is noticed that these displacements are very small under a load of 150 ton and nearly the same along the pile length for both soft and stiff clays.

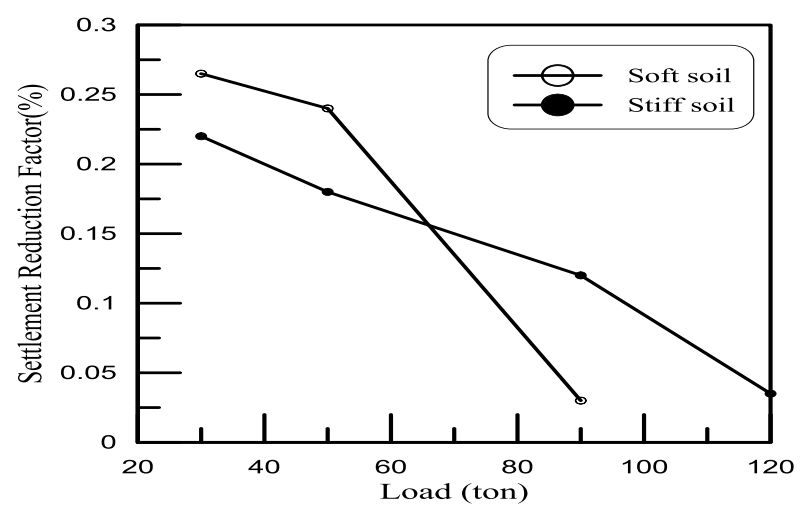

a. $\mathrm{L}=8 \mathrm{~m}, \mathrm{D}=1.0 \mathrm{~m}$

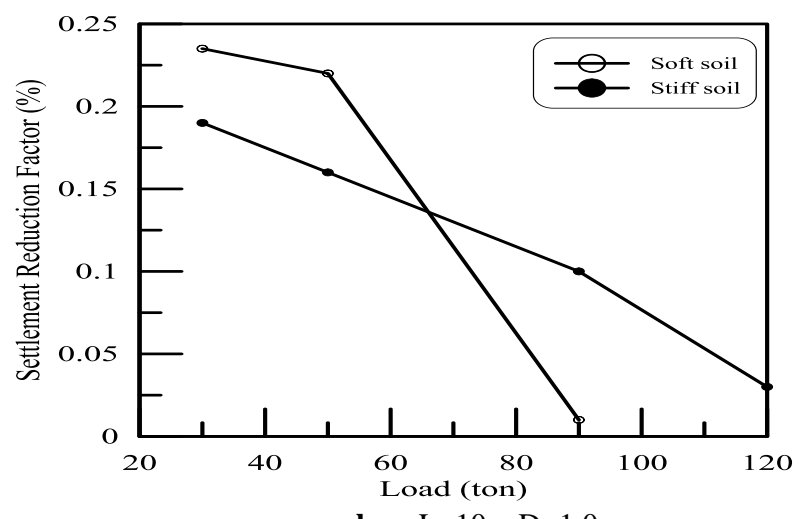

b. $\mathrm{L}=10 \mathrm{~m}, \mathrm{D}=1.0 \mathrm{~m}$

\section{a. Soft to medium soil}




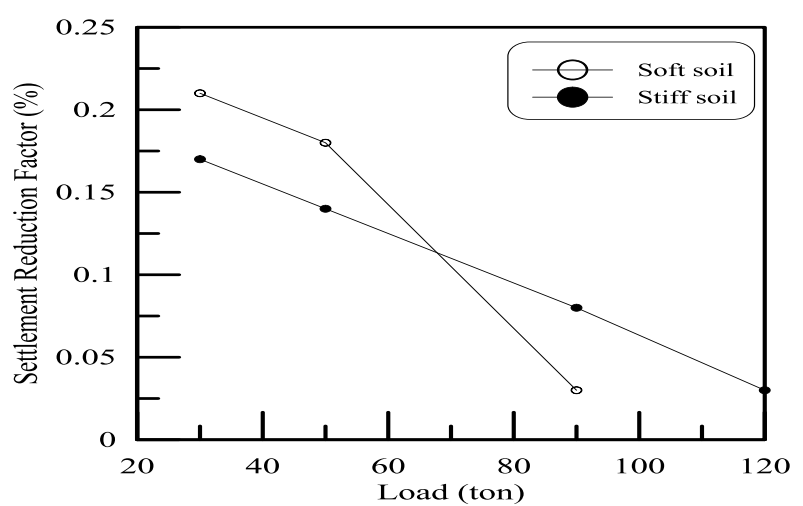

c. $\mathrm{L}=12 \mathrm{~m}, \mathrm{D}=1.0 \mathrm{~m}$

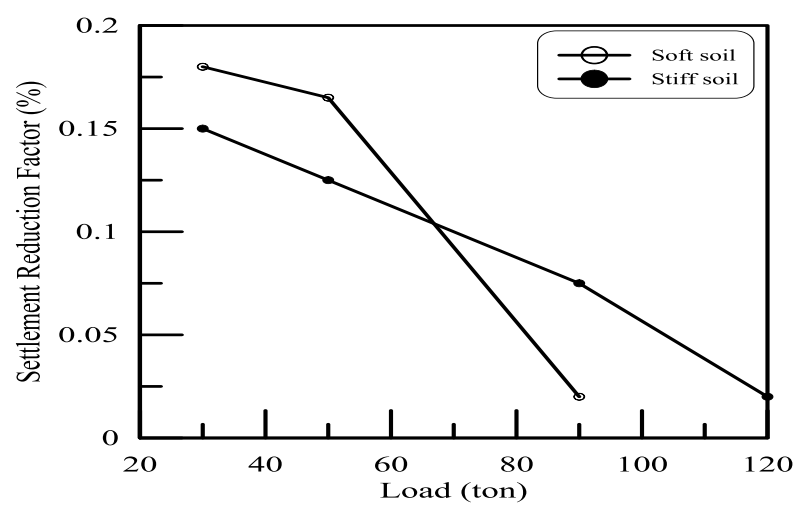

d. $\mathrm{L}=14 \mathrm{~m}, \mathrm{D}=1.0 \mathrm{~m}$

Fig. 5: Variation of the settlement reduction factor with load
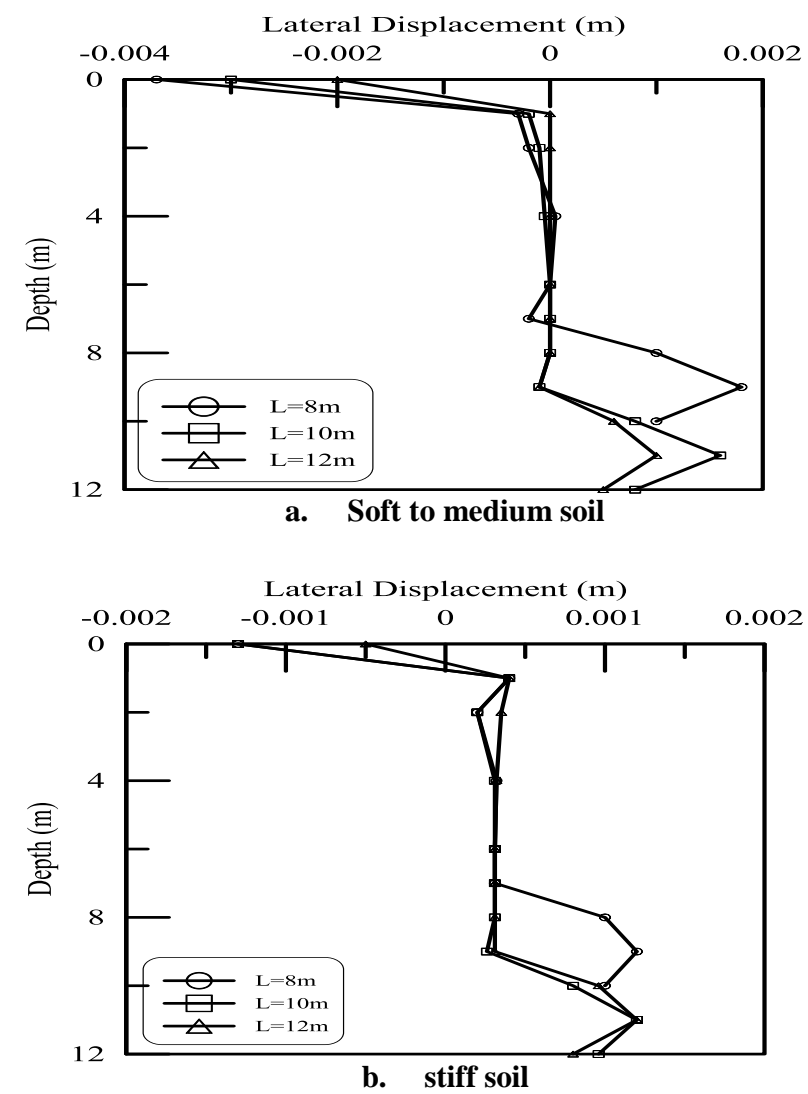

Fig. 6: Distribution of lateral displacement with depth $(D=1.0)$

\section{Conclusion}

1. In soft soil, it is found that the increase in the pile length from $(6 \mathrm{~m})$ to $(12 \mathrm{~m})$ an increase in the ultimate load in range (35- 60\%).

2. Increase the ultimate load of the pile with increasing diameter of pile for soft soil.

3. It is observed that the reduction factor of settlement with depth in both soft, medium and stiff clay is nearly control.

4. In soft soil, it is found the settelement reduction factor is greater than for the stiff clay.

5. An increase in bearing capacity of pile can be obtained when pile length is increased from $6 \mathrm{~m}$ to $12 \mathrm{~m}$.

\section{References}

[1] Ahmed, A.A. "Performance of Stone Columns in Soft Soils" Ph.D. Thesis, University of Baghdad (1998).

[2] Al-ani, M.A. "Solution of Problems of Piles in Clayey Soils by Finite Element Method" M.Sc. Thesis, University of Al-Mustansiria (2001).

[3] Desai, C.S. "Soil- Structure Introduction and Simulation Problems in Finite Element in Geomechanics" edited by Gudehus,G.John Wiley and Sons,P.P.209-250, (1977).

[4] Desai, C.S. , Zaman, M.M. Lightner, J.G. and Siriwardane, H.J. " Thin-Layre Element for Inter Faces and Joints" International Journal for Numerical and Analytical Method in Geomechanics ,Vol.8 , no. 1 , P.P. 19-43, (1984) .

[5] Focht, J.A. "Discussion to Paper by Coyle and Reese" Journal of the Soil Mechanics and Foundation Division ,ASCE, Vol.93, No.SM1, P.P. 133-138, (1967).

[6] Goodman, R.E. Taylor, R.L. and Brekke, T.L. "A model for the mechanics of Jointed Rock" Journal of Soil Mechanics and Foundation Engineering, London, Vol.2, P.P.35-39, (1968).

[7] Meyerhof, C. G. "Compaction of Sand and bearing Capacity of Piles", Journal of the Soil Mechanics and Foundation Division, ASCE, Vol. 85, No. SM6, P.P.1-29, (1959).

[8] Mindlin, R.D. "Force at a Point in The Interior of a Semi-Infinite Solid" Journal of the American Institute of Physics, vol.7, Np.2, P.P.205-223, (1936).

[9] Poulos, H.G. and Davis, E. H. "Pile Foundation Analysis and Design", John Wiley And Sons, New York, (1980)

[10] Poulos, H.G. "Analysis of Residual stress Effect in Piles" Journal of Geotechnical Engineering, ASCE, Vol.113, No.3, P.P.216-229, (1987).

[11] Smith, I.M. and Griffithes, D.V. "Programming The Finite Element Method", John Wiley and Sons, (1988).

[12] Yang, Z. and Jeremic, B. "Study of soil layering effects on lateral loading behavior of piles", Journal of Geotechnical Engineering, ASCE, Vol. 27, No.15, P.P.1255-1276, (2003).

[13] Ashour, M., Pilling, P. and Norris, G, Asce, M. "Lateral behavior of pile groups in layered soil", Journal of Geotechnical Engineering, ASCE, Vol. 130, No.6, (2004).

[14] Abu-Farsakh, M., "Finite Element Analysis of A Full-Scale Lateral Load Test on Batter Pile Group Foundatio", Ph.D. Thesis, Louisiana State university (2011).

[15] Toma, T." Nonlinear Finite Element Analysis of LaterallyLoaded Piles in Cohesive Sediments", Journal of Engineering and Technology, vol.28, No.2, (2010).

Eng. \& Tech. Journal, Vol. 28, No. 2, 2010Eng. \& Tech. Journal, Vol. 28, No. 2, 2010

Eng.\& Tech. Journal, Vol. 28, No. 2, 2010Eng. \& Tech. Journal, Vol. 28, No. 2, 2010 